\title{
Extrusion Parameters for Foaming of a $\beta$-Glucan Concentrate
}

\author{
Kristina Karlsson $^{1}\left[\right.$ [ Emanuel Larsson ${ }^{2} \cdot$ Niklas Lorén $^{2} \cdot$ Mats Stading $^{1,2} \cdot$ Mikael Rigdahl $^{1}$
}

Published online: 11 March 2019

(c) The Author(s) 2019

\begin{abstract}
Plastics is a group of materials commonly encountered on a daily basis by many people. They have enabled rapid, low-cost manufacturing of products with complicated geometries and have contributed to the weight reduction of heavy components, especially when produced into a foamed structure. Despite the many advantages of plastics, some drawbacks such as the often fossil-based raw-material and the extensive littering of the material in nature, where it is not degraded for a very long time, needs to be dealt with. One way to address at least one of the issues could be to use polymers from nature instead of fossil-based ones. Here, a $\beta$-glucan concentrate originating from barley was investigated. The concentrate was processed into a foam by hot-melt extrusion, and the processing window was established. The effect of different blowing agents was also investigated. Water or a combination of water and sodium bicarbonate were used as blowing agents, the latter apparently giving a more uniform pore structure. The porous structure of the foamed materials was characterized mainly by using a combination of confocal laser scanning microscope and image analysis. The density of the samples was estimated and found to be in a similar range as some polyurethane foams. A set of 3D parameters were also quantified on two selected samples using X-ray microtomography in combination with image analysis, where it was indicated that the porous structure had a pre-determined direction, which followed the direction of the extrusion process.
\end{abstract}

Keywords Hemicellulose $\cdot$ Starch $\cdot$ Extrusion $\cdot$ Image analysis $\cdot$ X-ray microtomography

\section{Introduction}

Plastics is the collective term used for a large number of polymeric materials which has become indispensable in our society during the past century. Plastics have contributed to the possibilities of weight reductions in the construction and transportation sectors and also to the reduction of waste of food in various packaging applications, they have enabled us to produce shapes and geometries which were unimaginable before and it has reduced production time and cost in the manufacturing industry. More recently, however, some negative side effects of our extensive use of fossil-based plastics has been brought to attention. In addition to an often fossil origin of the polymers, littering and leaching of small plastic

Kristina Karlsson

kristina.karlsson@chalmers.se

1 Department of Industrial and Materials Science, Chalmers University of Technology, Rännvägen 2A, 41296 Gothenburg, Sweden

2 Bioscience and Materials, Research Institutes of Sweden (RISE), PO Box 5401, 40229 Gothenburg, Sweden fractions to the environment, in combination with its nonbiodegradable nature, has proven to be a growing problem which must be solved [1-3]. One way to approach a solution could be to use natural or bio-based polymers.

One of the most important and commonly used techniques to produce a wide range of plastic products is hot-melt extrusion, or simply extrusion. The technique is continuous and by defining the processing parameters, the properties of the extrudate can be tailored. Extrusion is most commonly used to produce circular geometries such as tubes and cables, or flat sheets such as films and plates. But as already mentioned, extrusion is a versatile technique and can for example also be used in foaming applications. By adding a blowing agent, such as a liquid or a gas, the pressure builds up and hence the pressure drop created over the nozzle can be used to tailor the porosity of the material. Also the choice of blowing agent, or a combination of different ones, can be used as a method of controlling the pore size distribution and the connectivity.

The polymeric material in this study originated from a specific fraction of barley. The material is a combination of several components and it is generally referred to as a 
dietary fibre, here it will be referred to as a $\beta$-glucan concentrate. $\beta$-glucan is a hemicellulose, and is the most common non-starch component in the cell wall of cereal grains such as oat and barley [4]. The material is water soluble and its structure is based on a glucose chain connected by $\beta-(1 \rightarrow 3)$ and $\beta$-(1 $\rightarrow 4)$-linkages. When working with natural polymers the consideration of competition for food production has to be taken into account. Although $\beta$-glucan is used commercially in some food products, mainly with the aim of lowering the low-density lipoprotein (LDL) cholesterol in the blood [5-7], this $\beta$-glucan commonly originates from oat. The majority of the barley $\beta$-glucan is used as animal feed [4].

The rheological properties of the water-containing $\beta$-glucan concentrate at high temperatures in both shear and extension were examined previously. These properties were found to be in the same range as some conventional plastics and it was concluded that from a rheological point of view, the material would be suitable for extrusion [8], which is the focus of the present study.

The aim of the present work was to indicate a processing window for extrusion foaming for the $\beta$-glucan concentrate in terms of processing parameters and water content. The foaming ability of the material was described in terms of the porosity (density) and the pore size distribution. The water in the $\beta$-glucan concentrate acted as a blowing agent (in the form of steam at higher temperatures), but the effect of additional blowing agents, such as sodium bicarbonate and citric acid, on the foaming behaviour was also investigated.

\section{Experimental}

\section{Materials}

For the extrusion experiments, a $\beta$-glucan concentrate by the name "Lyckeby beta-glucan fiber DF 25" was used. The concentrate was kindly supplied by Lyckeby Starch $\mathrm{AB}$ (Sweden). The $\beta$-glucan originated from barley and the composition of the concentrate, as given by the supplier, is shown in Table 1.

Sodium bicarbonate was purchased from VWR International (USA) and citric acid from Merck (Germany) and they were used as received.

\section{Methods}

\section{Preparation of the Material}

The $\beta$-glucan concentrate in powder form was mixed with water and in some cases sodium bicarbonate and citric acid for about $2 \mathrm{~min}$ in a kitchen food processor (Bosch, Germany). As mentioned, these three components were the
Table 1 Information about the composition of the $\beta$-glucan concentrate was received from the manufacturer

\begin{tabular}{lll}
\hline Component & $\begin{array}{l}\text { Amount } \\
\text { [weight-\%] }\end{array}$ & Method \\
\hline $\begin{array}{l}\text { Dietary fibre } \\
\text {-whereof } \beta \text {-glucan }\end{array}$ & $40-45$ & AOAC 985.29 \\
Starch & $30-35$ & AOAC 995.16 \\
Protein & $10-12$ & AOAC 996.11 ${\text { Kjeldahl titration }{ }^{\mathrm{d}} \mathrm{N}^{*} 6,25 \text { acc. }}^{\mathrm{a}}$ \\
Fat & 3 & (EC) No $152 / 2009^{\mathrm{e}}$ \\
\hline
\end{tabular}

$\beta$-Glucan constitutes about $25 \%$ of the weight of the product. The component denoted dietary fibre contains $\beta$-glucan, arabinoxylan, cellulose and some proteins

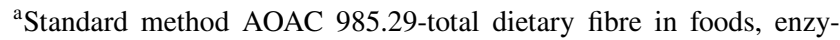
matic-gravimetric method, http://www.eoma.aoac.org/methods/info. asp?ID $=14700$

${ }^{\mathrm{b}}$ Standard method AOAC 995.16- beta-D-glucan in barley and oats, streamlined enzymatic method, http://www.eoma.aoac.org/methods/ info.asp?ID $=21738$

${ }^{\mathrm{c}}$ Standard method AOAC 966.11-starch (total) in cereal products, amyloglucosidase-alpha-amylase method, http://www.eoma.aoac.org/ methods/info.asp?ID=27501

${ }^{\mathrm{d} K j e l d a h l ~ t i t r a t i o n: ~ K j e l d a h l, ~ J . ~(1883) ~ " N e u e ~ M e t h o d e ~ z u r ~ B e s t i m-~}$ mung des Stickstoffs in organischen Körpern" (New method for the determination of nitrogen in organic substances), Zeitschrift für analytische Chemie, 22 (1) p. 366-383

${ }^{\mathrm{e}}$ Commission regulation (EC) No 152/2009 of 27 January 2009 laying down the methods of sampling and analysis for the official control of feed, OJ L 54, 26.2.2009, p. 1-130, http://eur-lex.europa.eu/legal -content/EN/TXT/?uri=CELEX:32009R0152

blowing agents. The added amount of water was $30 \%$ by weight to the $\beta$-glucan concentrate weight for batch 1 and 2 , and the actual water content was determined gravimetrically in the pellets before extrusion. Sodium bicarbonate, $5 \%$ by weight based on the weight of the $\beta$-glucan concentrate (not considering the water weight), was added in some cases. For the samples containing citric acid, an equal amount (by weight) of citric acid as sodium bicarbonate was added. Here, the added water content was adjusted and $30 \mathrm{wt}-\%$ was added based on the total weight of all the other three components. Table 2 shows a summary of the content of the three different batches of the material produced. The water content in the $\beta$-glucan concentrate in powder form, measured before any treatment, was about $7 \mathrm{wt}-\%$.

When referring to a material from one of the different mixing conditions, it will be denoted B1, B2 or B3.

\section{Extrusion of Pellets}

The prepared materials were extruded using a single-screw extruder (Brabender stand-alone extruder, E 19/25 D, Germany). A compression screw (3:1 compression ratio) with the diameter $D=19 \mathrm{~mm}$ and the length $L=25 D$ was used 
Table 2 A summary of the different batches of material produced using three different mixing compositions

\begin{tabular}{ll}
\hline Batch & Composition \\
\hline B1 & $\beta$-glucan contentrate \\
& 30 wt- $\%$ water \\
B2 & $\beta$-glucan contentrate \\
& 5 wt-\% sodium bicarbonate \\
& 30 wt- $\%$ water \\
B3 & $\beta$-glucan contentrate \\
& 5 wt- $\%$ sodium bicarbonate \\
& Citric acid (equal amount \\
& to sodium bicarbonate, by \\
& weight) \\
& 30 wt- $\%$ water \\
\hline
\end{tabular}

to compact and properly mix the components together into a cohesive string. The extruder had four individually controlled heating zones, three in the extruder barrel and one in the die. For the pellet production the temperatures were set to $30{ }^{\circ} \mathrm{C}$ in the first (feeding) zone and $60{ }^{\circ} \mathrm{C}$ in the remaining three. A die with a diameter of $3 \mathrm{~mm}$ and a rotational speed of $80 \mathrm{rpm}$ was used for the extrusion. The extruded strings were then pelletized with a granulator (SG $10 \mathrm{Ni}$ Dreher, Germany) and the pellets were kept in air tight plastic bags and frozen, in order to preserve their moisture content, until further used.

\section{Extrusion Foaming}

The pellets were defrosted and the samples were taken for analysis of their moisture content before the extrusion foaming. The diameter of the die was kept at $2 \mathrm{~mm}$ throughout the experiments. The temperatures of the three heating zones in the barrel were set to 70,85 and $105{ }^{\circ} \mathrm{C}$ respectively from the feeding zone to the final zone before the die. The temperature of the die zone was varied between 110, 130 and $150{ }^{\circ} \mathrm{C}$ and the screw rotational speed was varied between 30, 80 and $120 \mathrm{rpm}$ as shown in Table 3. Samples were taken at the different test conditions and the moisture content of the extrudates was determined by placing the samples in an oven at $105^{\circ} \mathrm{C}$ while recording the weight until it was stable.

The sample notations in Table 3 refer in the following to the produced foams. After the extrusion, samples were collected and stored in a freezer, before further evaluation by microscopy and X-ray microtomography.

\section{Microscopy}

The extruded $\beta$-glucan foamed strings were cut into about $2 \mathrm{~mm}$ long pieces with a surgical-stainless steel blade and placed flat with the cross-sectional surface down on a thin glass cover. At least 8 samples of each direction, were used for every experiment. A Leica TCS SP2 (Leica Ltd,
Table 3 Extrusion parameters used during the foaming experiments

\begin{tabular}{llc}
\hline Sample notation & Die temperature $\left({ }^{\circ} \mathrm{C}\right)$ & $\begin{array}{c}\text { Rotational } \\
\text { speed }(\mathrm{rpm})\end{array}$ \\
\hline B1-1 & 110 & 30 \\
B1-2 & 110 & 80 \\
B1-3 & 110 & 120 \\
B1-4 & 130 & 30 \\
B1-5 & 130 & 80 \\
B1-6 & 130 & 120 \\
B1-7 & 150 & 30 \\
B1-8 & 150 & 80 \\
B1-9 & 150 & 120 \\
B2-1 & 110 & 30 \\
B2-2 & 110 & 80 \\
B2-3 & 110 & 120 \\
B2-4 & 130 & 30 \\
B2-5 & 130 & 80 \\
B2-6 & 130 & 120 \\
B2-7 & 150 & 30 \\
B2-8 & 150 & 80 \\
B2-9 & 150 & 120 \\
B3-1 & 130 & 80 \\
B3-2 & 150 & 80 \\
B3-3 & 170 & 80 \\
\hline
\end{tabular}

The sample notations refer to produced foams

Heidelberg, Germany) confocal laser scanning microscope (CLSM) was used to obtain micrographs of the samples. The magnification used was $5 \mathrm{X}$ and the the numerical aperture of the objective was 0.12 . The image format was $1024 \times 1024$ pixels $^{2}$ and the pixel size was $2.9 \mu \mathrm{m}$. Due to the auto-fluorescent nature of the samples, no additional staining was required.

\section{Image Analysis}

The images from the CLSM were post-processed using the image processing package Fiji, distributed by ImageJ2 [9]. First, a median filter was applied, thereafter the image was made binary. As a last step an open- and a closing-operation was performed to combine pores and eliminate very small elements.

The binary images were then evaluated by an automatic measuring program using the software AxioVision E64 Rel. 4.9.1 by Carl Zeiss (Germany). In the program, all pores are identified and their diameter measured. All elements containing less than 100 pixels were excluded. By evaluating the average diameter of each sample, the expansion of the extrudate area, as compared to the die area, could be calculated. The reported number of pores refers to an average number of pores per cross-section examined. The average 
pore area, which in a sense can be regarded as the porosity, is calculated as a percentage of the pore area to the total crosssection sample area. All numbers reported on are averages from 5 cross-sectional images examined.

\section{Density Evaluation}

The apparent density of the foamed samples was evaluated by weighing samples and approximating their volume. Pieces were cut from the extruded strings and the weight was recorded using a balance (Mettler Toledo AG285, USA). The length of the cut piece was measured by calipers and the diameter was taken as the average diameters of the samples from the microscopy study.

\section{X-Ray Microtomography}

$\mathrm{X}$-ray microtomography a is non-destructive $3 \mathrm{D}$ imaging technique, which was hereby applied on only two samples (B1-6 and B2-6, i.e. samples produced at a temperature of $130{ }^{\circ} \mathrm{C}$ and with the rotational speed $120 \mathrm{rpm}$, with and without sodium bicarbonate, respectively) for visualizating and quantifying certain extrusion-related properties and sample characteristics occurring in 3D. The samples were scanned using a ZEISS Xradia 520 Versa system (Carl Zeiss, Germany) at the 4D Imaging Lab at Lund University. The scanning parameters were the following, source-voltage: $50 \mathrm{kV}$, source power: $4 \mathrm{~W}$, exposure time: $1 \mathrm{~s}$, number of projections: 1601 , $4 \mathrm{x}$ optic with a sample-to-detector distance: $13.04 \mathrm{~mm}$, Field-of-View (FOV): $3.65 \times 3.65 \mathrm{~mm}^{2}$ and effective isotropic pixel size: $3.6 \mu \mathrm{m}$. The linear X-ray attenuation coefficient (LAC) $\left[\mathrm{cm}^{-1}\right]$ was reconstructed on a 16-bit grey level scale using the manufacturer-supplied reconstruction software.

\section{Quantitative 3D Image Analysis}

The reconstructed slices contained three well-separated peaks, associated to air (weekly absorbing part), $\beta$-glucan (average absorbing part) and a high-absorbing part (likely associated with residual cell wall material), which were separated using standard grey-level thresholding in Fiji [9], whereinafter they were analysed using the Pore3D software package for quantitative analysis of porous media [10]. A test for finding the Representative Volume of Interest (RVI) [11] revealed an optimal VOI size of $1.26 \times 1.26 \times 1.26 \mathrm{~mm}^{3}$. Hereinafter, two representative VOIs (Volume Of Interest) per sample were chosen inside the sample, far away from sample edges. The extracted VOIs were used to quantify a set of descriptive 3D image parameters, such as Percentage Volume (\%), Isotropy index (-), Connectivity Density (Conn.D) $\left(\mathrm{mm}^{-3}\right)$, which provides a quantitative number on how well-connected a structure is, and pore sizes $(\mu \mathrm{m})$, as quantified by inscribing virtual 'blobs' and quantifiying their diameter, as further described in [10, 12].

\section{Visualization}

All 3D volume renderings of both the entire samples and extracted VOIs were performed using the Drishti package for visualization of volumetric datasets [13].

\section{Results and Comments}

\section{Moisture Content}

The moisture content of the pellets was measured after the pelletizing, freezing and defrosting steps. The moisture content of each sample was also determined after the extrusion foaming. All the measured values are given in Table 4.

The reason for the quite large water content present in the samples even after extrusion is due to the hygroscopic nature of the material. As already mentioned, the measured water content in the $\beta$-glucan concentrate in powder form, before any treatment, was about $7 \mathrm{wt}-\%$.

The moisture content was reduced with increasing temperature and residence time in the extruder (as was expected). The only exceptions were observed for specimens B2-1 to B2-3 for which the water content actually decreased with increasing rotational speed of the extruder screw. The difference is however not large and can probably be attributed to experimental scatter. A substantial amount of water (around 20\%) was still at hand in most

Table 4 The measured moisture content of the extrudates which contained only water (B1) as the blowing agent, the extrudates which contained water and sodium bicarbonate (B2) as the blowing agent and the extrudates which contained, water, sodium bicarbonate and citric acid (B3) as the blowing agent

\begin{tabular}{llllll}
\hline $\begin{array}{l}\text { Sample } \\
\text { B1 }\end{array}$ & $\begin{array}{l}\text { Moisture } \\
\text { content } \\
{[\%]}\end{array}$ & $\begin{array}{l}\text { Sample } \\
\text { B2 }\end{array}$ & $\begin{array}{l}\text { Moisture } \\
\text { content } \\
{[\%]}\end{array}$ & $\begin{array}{l}\text { Sample } \\
\text { B3 }\end{array}$ & $\begin{array}{l}\text { Moisture } \\
\text { content } \\
{[\%]}\end{array}$ \\
\hline $\begin{array}{l}\text { Pellets } \\
\text { before } \\
\text { extru- }\end{array}$ & 25.7 & $\begin{array}{l}\text { Pellets } \\
\text { before } \\
\text { sion }\end{array}$ & \multicolumn{5}{c}{$\begin{array}{l}\text { extru- } \\
\text { sion }\end{array}$} & & - & - \\
B1-1 & 22.6 & B2-1 & 23.4 & B3-1 & 18.2 \\
B1-2 & 22.6 & B2-2 & 22.0 & B3-2 & 17.0 \\
B1-3 & 22.5 & B2-3 & 21.6 & B3-3 & 16.7 \\
B1-4 & 21.3 & B2-4 & 19.5 & - & - \\
B1-5 & 22.1 & B2-5 & 20.6 & - & - \\
B1-6 & 22.1 & B2-6 & 20.4 & - & - \\
B1-7 & 19.7 & B2-7 & 18.2 & - & - \\
B1-8 & 20.6 & B2-8 & 19.6 & - & - \\
B1-9 & 19.8 & B2-9 & 20.5 & - & - \\
\hline
\end{tabular}


of the foams after the extrusion. The water content is however expected to decrease to about $7 \%$ (the water content of the $\beta$-glucan concentrate at room conditions), if let in the air to dry. The water content of the pellets before extrusion was unfortunately not recorded for the B3 pellets.

\section{Microscopical Studies of the Foamed Structure}

A significant difference in both the degree of expansion and the porosity could be observed when increasing the temperature and rotational speed of the extruder screw for batch 1 and 2 (B1 and B2), see Figs. 1 and 2. For batch 3 (B3), the differences were less pronounced, here only the temperature was changed, see Fig. 3.

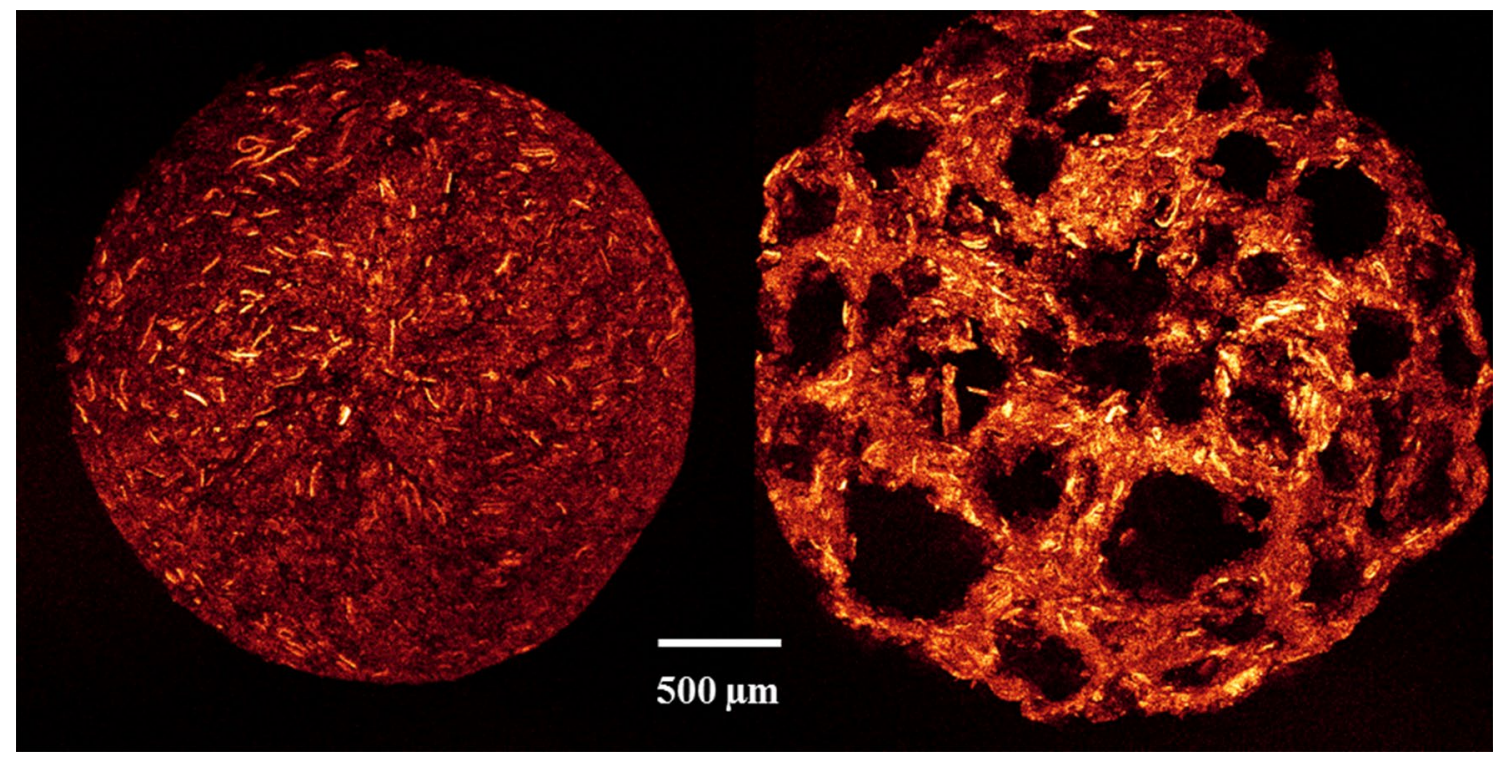

Fig. 1 CLSM micrographs of extruded foams. To the left: sample produced using material and processing parameters B1-1. To the right: sample produced using material and processing parameters B1-7

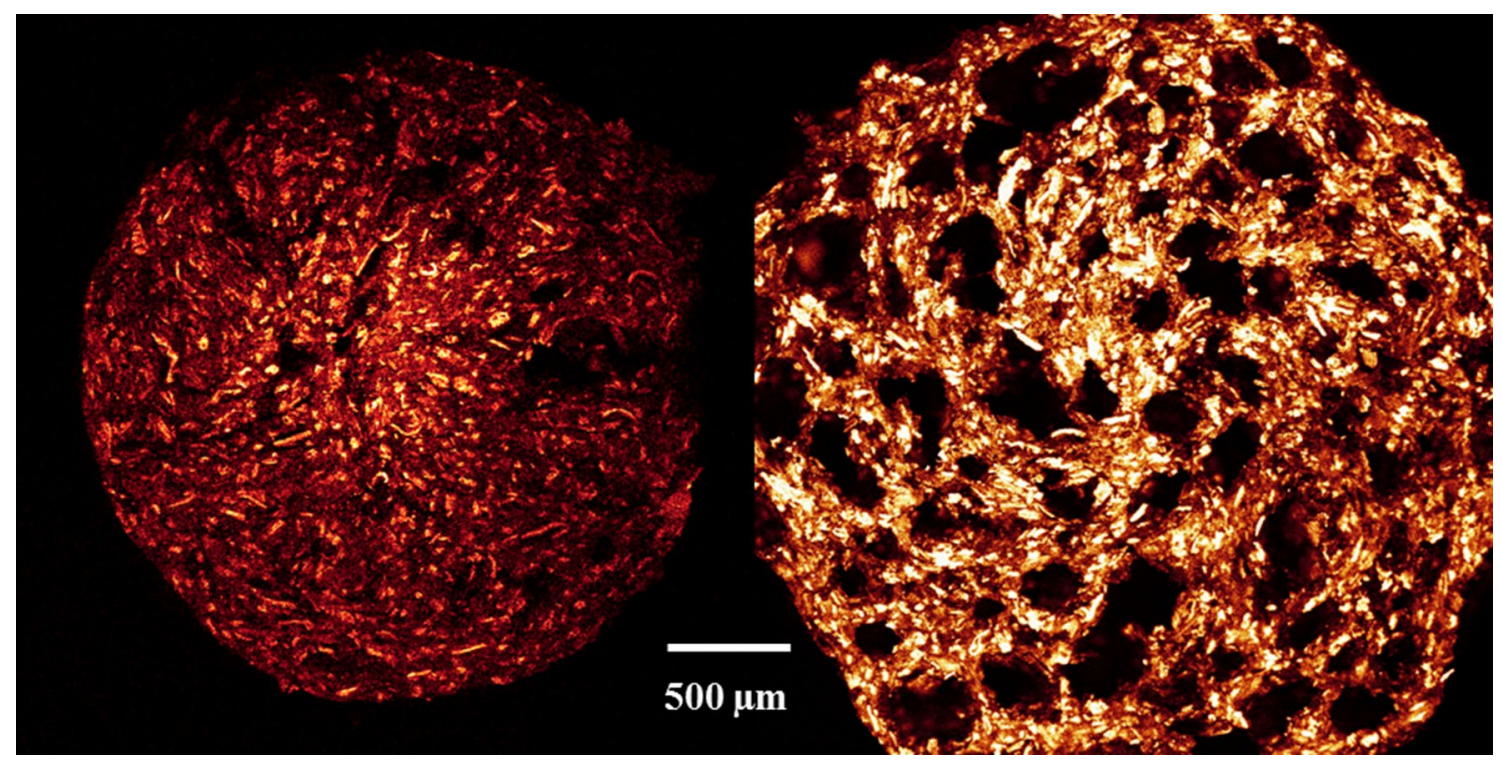

Fig. 2 CLSM micrographs of extruded foams. To the left: sample produced using material and processing parameters B2-1. To the right: sample produced using material and processing parameters B2-8 


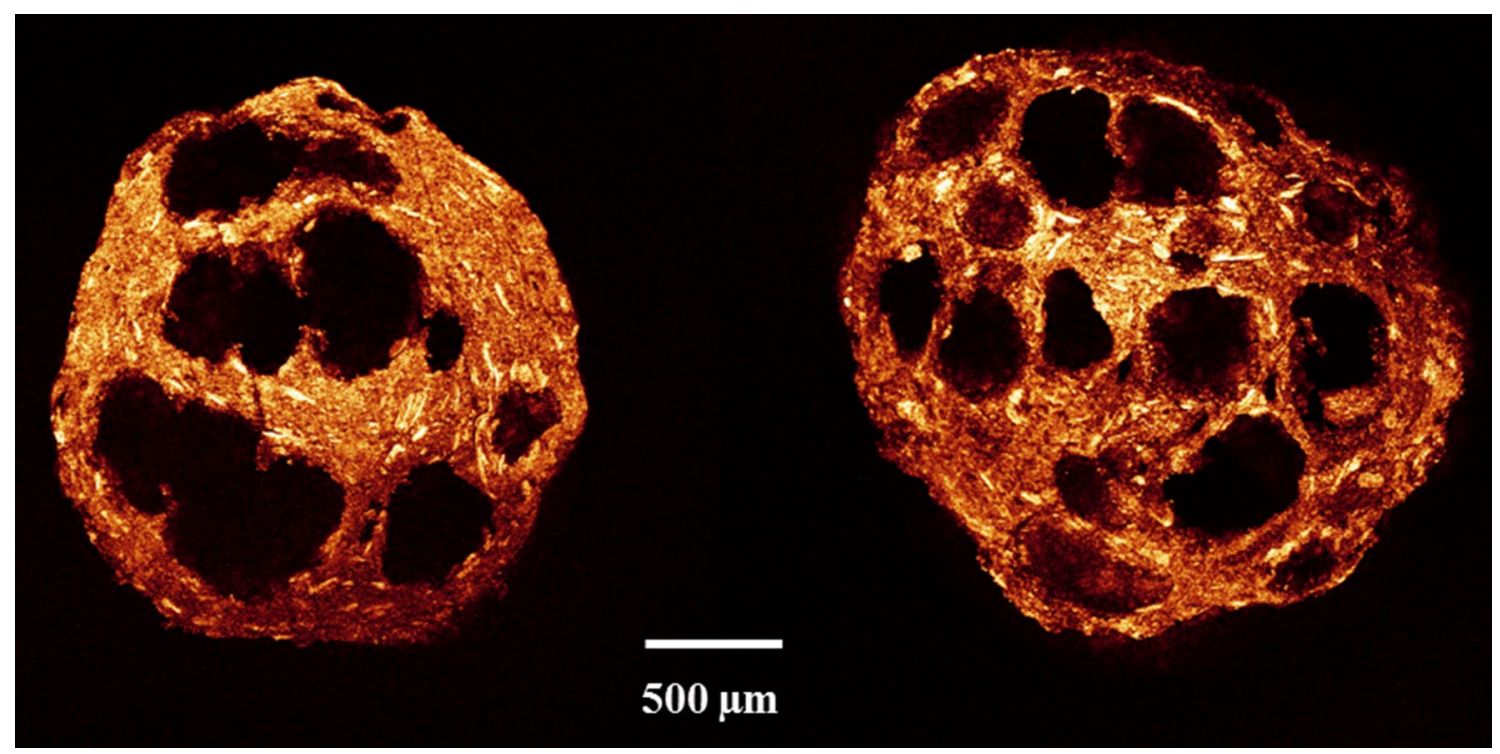

Fig. 3 CLSM micrographs of extruded foams. To the left: sample produced using material and processing parameters B3-1. To the right: sample produced using material and processing parameters B3-3

Some visual differences in foaming behaviour could be observed between batch 1 and 2 without further analyses. Even for the lowest temperature and rotational speed, samples from B2 exhibited some (although not very uniform) larger hollow areas, which could indicate the beginning of pore formation. Also for the higher temperatures and rotational speeds, a quite distinct difference can be observed between the two batches. Samples from batch 2, which also contained sodium bicarbonate as blowing agent, displayed a more uniform pore size distribution, often with more and smaller pores.

The foamed samples produced from batch 3 did not expand as much as samples from batch 1 or 2, see Fig. 3 . The pellets produced in the mixing step felt "dryer" and rougher than the pellets from the other two batches. Despite that the water content was increased for batch 3 , the addition of the citric acid seemed to influence the properties of the compound, making it less plasticised. This likely hindered the expansion during the extrusion and even at higher temperatures; samples from batch 3 did not expand to a much larger diameter than the die. However, a slight increase in expansion was observed with the highest die temperature $\left(170{ }^{\circ} \mathrm{C}\right)$.

\section{Image Analysis of the Micrographs}

Image analysis was applied to the micrographs obtained with CLSM and the results are shown in Table 5.

Some clear trends can be seen; the expansion increased both with increasing temperature and with increasing rotational speed of the screw. This is exemplified at the lowest temperature $\left(110{ }^{\circ} \mathrm{C}\right)$ in case of specimens $\mathrm{B} 1-1$ to $\mathrm{B} 1-3$ and B2-1 to B2-3 for which the expansion increased by an increasing rotational speed which is a consequence of the larger pressure drop over the die. Among the two batches, the $\mathrm{B} 2$ samples expanded more at the same processing conditions. For the middle temperature, $130{ }^{\circ} \mathrm{C}$ (B1-4 to B1-6, B2-4 to B2-6 and B3-1), a similar trend can be observed. The expansion at the lowest rotational speed, at the higher temperature, was lower than with the fastest speed at the lower temperature, i. e. $118 \%$ expansion at $130{ }^{\circ} \mathrm{C}$ and $30 \mathrm{rpm}$ as compared to $179 \%$ at $110{ }^{\circ} \mathrm{C}$ and $120 \mathrm{rpm}$ (B2-4 and B2-3, respectively). In a sense the rotational speed seems to be more important than the temperature at this point. At $150{ }^{\circ} \mathrm{C}$ (B1-7 to B1-9, B2-7 to B2-9 and B3-2), the same behaviour is observed again. Also here, samples from batch 2 (with sodium bicarbonate) performed better than specimens from B1 and B3 and the greatest expansion of $197 \%$ is observed for sample B2-9, with a temperature of $150{ }^{\circ} \mathrm{C}$ and a rotational speed of $120 \mathrm{rpm}$. Overall, samples from batch 3 expanded poorly and the results were significantly worse than for the samples from batch 1 , containing only water. However, an increase in temperature still increased the expansion somewhat.

Looking at the number of pores in the samples, the trend is not as obvious. In general, the samples with a greater expansion exhibited a greater number of pores, but this is not true in all cases. It is noteworthy that the range of number of pores was much smaller for batch 2 than for batch 1 . The perceived increased uniformity in the pore size distribution that was seen in the CLSM micrographs is thus confirmed by the image analysis. Furthermore, the often lower standard 
Table 5 The expansion (\%) in comparison to the die diameter, the average number of pores per cross-sectional image taken, the average pore diameter $(\mu \mathrm{m})$ and the pore volume per cross-sectional image taken (\%) for the samples as obtained from the image analysis of the micrographs

\begin{tabular}{lccll}
\hline Sample & Expansion $(\%)$ & $\begin{array}{l}\text { Average number of pores } \\
\text { per cross-sectional image }\end{array}$ & $\begin{array}{l}\text { Average pore } \\
\text { diameter }(\mu \mathrm{m})\end{array}$ & $\begin{array}{l}\text { Average pore area per } \\
\text { cross-sectional image } \\
(\%)\end{array}$ \\
\hline B1-1 & $59 \pm 2$ & $114 \pm 31$ & $73 \pm 6$ & $13 \pm 1$ \\
B1-2 & $74 \pm 2$ & $162 \pm 25$ & $75 \pm 4$ & $21 \pm 1$ \\
B1-3 & $85 \pm 5$ & $171 \pm 17$ & $77 \pm 5$ & $25 \pm 2$ \\
B1-4 & $69 \pm 14$ & $94 \pm 13$ & $85 \pm 17$ & $23 \pm 5$ \\
B1-5 & $79 \pm 5$ & $86 \pm 14$ & $91 \pm 10$ & $26 \pm 2$ \\
B1-6 & $115 \pm 5$ & $135 \pm 12$ & $87 \pm 6$ & $38 \pm 4$ \\
B1-7 & $117 \pm 7$ & $91 \pm 11$ & $127 \pm 7$ & $41 \pm 3$ \\
B1-8 & $131 \pm 13$ & $86 \pm 11$ & $129 \pm 4$ & $41 \pm 2$ \\
B1-9 & $159 \pm 11$ & $124 \pm 32$ & $109 \pm 16$ & $42 \pm 2$ \\
B2-1 & $68 \pm 3$ & $138 \pm 12$ & $72 \pm 3$ & $19 \pm 1$ \\
B2-2 & $117 \pm 10$ & $139 \pm 13$ & $101 \pm 9$ & $39 \pm 3$ \\
B2-3 & $179 \pm 11$ & $136 \pm 16$ & $118 \pm 14$ & $44 \pm 1$ \\
B2-4 & $118 \pm 14$ & $130 \pm 19$ & $104 \pm 9$ & $38 \pm 2$ \\
B2-5 & $143 \pm 6$ & $147 \pm 14$ & $106 \pm 7$ & $39 \pm 1$ \\
B2-6 & $193 \pm 10$ & $136 \pm 9$ & $134 \pm 11$ & $44 \pm 1$ \\
B2-7 & $106 \pm 9$ & $163 \pm 17$ & $101 \pm 4$ & $35 \pm 1$ \\
B2-8 & $176 \pm 6$ & $152 \pm 14$ & $131 \pm 6$ & $43 \pm 1$ \\
B2-9 & $197 \pm 6$ & $161 \pm 9$ & $134 \pm 5$ & $43 \pm 1$ \\
B3-1 & $7 \pm 9$ & $48 \pm 11$ & $122 \pm 26$ & $39 \pm 4$ \\
B3-2 & $19 \pm 12$ & $62 \pm 17$ & $122 \pm 24$ & $47 \pm 6$ \\
B3-3 & $22 \pm 6$ & $65 \pm 16$ & $125 \pm 26$ & $49 \pm 5$ \\
\hline
\end{tabular}

The standard deviation is presented next to each value deviations in the number of pores for sample B2 indicates a more uniform distribution of pores.

One interesting observation is that for samples from batch 1 , the pore diameters and the pore volumes appeared to increase with an increase in processing temperature. For samples from batch 2 however, the increase in diameter and volume of the pores tended to be more related to the rotational speed of the screw than to the processing temperature. For samples from batch 3, the size and volume of the pores was quite uniform and only increased slightly with increasing temperature.

\section{Image Analysis of Microtomography Data Sets}

The 3D-renderings of the entire and cut samples B1-6 and B2-6 are shown in Fig. 4; the specimens were produced at the same processing conditions but with (B2-6) and without (B1-6) the sodium bicarbonate as the additional blowing agent. For clarity, only one quarter of the volume element is shown here. Visually it can be seen that the use of sodium bicarbonate as for B2-6 resulted in a more porous sample, with smaller pores and a more uniform pore structure, than for B1-6. This observation is also confirmed by the quantitative $3 \mathrm{D}$ image analysis as reported in Table 6, which was performed on the extracted
VOIs, as exemplified in Fig. 5 in the case of B2-6. More in detail, sample B2-6 was roughly $20 \%$ more porous than sample B1-6, as highlighted by the Percentage Volume parameter. The opposite effect was naturally seen for the $\beta$-glucan part.

An isotropy index close to 1 indicates that there is "no preferred direction', and consequently a value closer to 0 indicates that there is 'a preferred direction'.The isotropy index reported in Table 6 confirms that there is a slight preferred direction of the pores and the $\beta$-glucan phase in both samples. This observation is also confirmed by Fig. 4, where it is shown that the preferred direction is aligned with the extrusion direction of the sample.

The quantified connectivity density parameter in Table 6 , reveals that both the porous and $\beta$-glucan parts were more connected in sample B1-6 than in sample B2-6. The connectivity of the structure is visually exemplified in Fig. 5e in the case of sample B2-6. It is also clear from the analysis that B2-6 has many more isolated non-connected skeleton branches (marked in orange in Fig. 5e), which were excluded from the calculation of the connectivity density. Table 6 also shows indications of that the maximum pore size was larger in sample B1-6 than in sample B2-6. 
Fig. 4 3D volume reconstruction of the extruded $\beta$-glucan concentrate after applying virtual cut planes. For A, produced without sodium bicarbonate, the total sample height was roughly $3.6 \mathrm{~mm}$ and the entire sample diameter on average $2.6 \mathrm{~mm}$ (3.2 $\mathrm{mm}$ on the upper top in the widest direction). For B, produced with sodium bicarbonate, the total sample height was roughly $3.6 \mathrm{~mm}$ and the sample diameter on average $3.3 \mathrm{~mm}$. The high absorbing part is seen (in blue). (Color figure online)
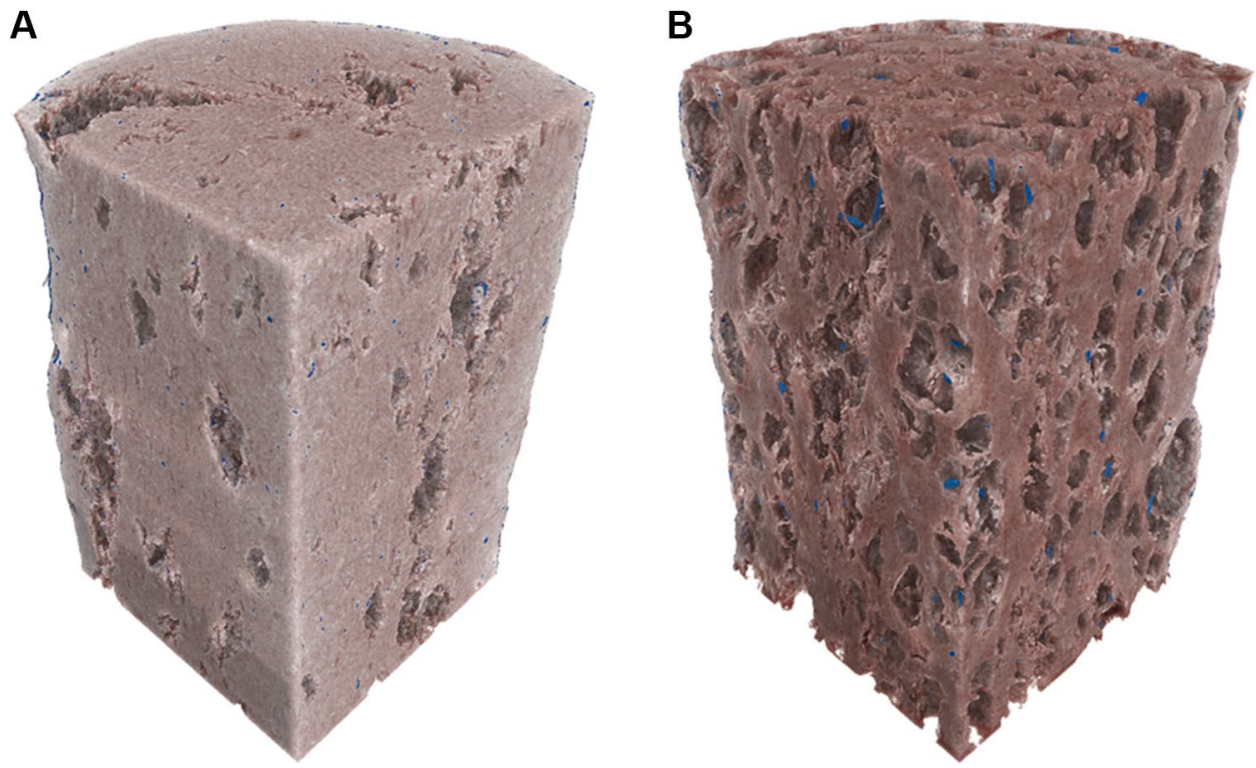

Table 6 Extracted image descriptors (mean values and standard deviations) for the pores and the $\beta$-glucan phase for B1-6 and B2-6, obtained by applying quantitative $3 \mathrm{D}$ image analysis on selected VOIs, with a representative VOI size of $1.26 \times 1.26 \times 1.26 \mathrm{~mm}^{3}$

\begin{tabular}{llcl}
\hline Quantified parameter & Sample & Porous part & $\beta$-glucan \\
\hline Percentage volume (\%) & B1-6 & $19.7 \pm 3.6$ & $80.0 \pm 3.6$ \\
& B2-6 & $39.6 \pm 2.2$ & $60.2 \pm 2.1$ \\
Isotropy index (-) & B1-6 & $0.78 \pm 0.04$ & $0.81 \pm 0.05$ \\
& B2-6 & $0.74 \pm 0.05$ & $0.75 \pm 0.03$ \\
Connectivity density & B1-6 & $12 \pm 5$ & $394 \pm 8$ \\
$\left(\right.$ Conn.D) $\left(\mathrm{mm}^{-3}\right)$ & B2-6 & $4 \pm 1$ & $287 \pm 21$ \\
Maximum pore size $(\mu \mathrm{m})$ & B1-6 & $119 \pm 64$ & - \\
& B2-6 & $111 \pm 46$ & - \\
\hline
\end{tabular}

\section{Density of the Foamed Extrudates}

Density is a very important property when it comes to characterizing foams. The apparent densities of the extruded foams are shown in Fig. 6. The density values were compared to an un-foamed (solid) sample consisting of only $\beta$-glucan concentrate mixed with water, having a total water content of $9 \%$. The density of the un-foamed sample was $1.3 \mathrm{~g} / \mathrm{cm}^{3}$ which can be compared to sample B1-2 having the highest density of the foamed materials, being around $1.2 \mathrm{~g} /$ $\mathrm{cm}^{3}$ and sample B2-9 and sample B3-3 having the lowest values in the range of $0.55 \mathrm{~g} / \mathrm{cm}^{3}$. These values are rather high compared to expanded polystyrene densities which usually attain very low values in the range of $0.015-0.030 \mathrm{~g} / \mathrm{cm}^{3}$ [14]. However, they are comparable to some reported values for polyurethane foams which are in the range of $0.1-0.7 \mathrm{~g} /$ $\mathrm{cm}^{3}$, depending on the grade used [15]. The density of the non-porous sample corresponded quite well to reported densities of other hemicelluloses which ranged from 1.37 to $1.52 \mathrm{~g} / \mathrm{cm}^{3}[16,17]$. There was also a clear trend within each batch where the density decreased from sample B1-1 to B1-9, from B2-1 to B2-9 and from B3-1 to B3-3; i. e. with increasing processing temperature. This trend correlates with the porosity measurements, since the density is expected to decrease with an increased porosity.

\section{Discussion}

From the microscopy results, followed by the subsequent analysis of the images taken, it was clear that the foamed samples produced from batch 2 exhibited the greatest expansion and also a more uniform spatial distribution of the pores as well as a more uniform pore size distribution. Batch 1, which only contained water as the blowing agent, also performed reasonably well, although the expansion was about $10 \%$ lower than for samples from batch 2 and the pore size distribution was somewhat broader. The observation that the pores follow the extrusion direction, as quantified by isotropy index based on the $\mu \mathrm{CT}$ images, is also in line with the observations reported in [18], where $\mu \mathrm{CT}$ was used to show that cellulose fibers in poly(lactic acid)/wood fibers bio-composites followed the orientation of the flow direction of the melt, which was correlated to the geometry of the die and the pressure in the extruder.

Samples produced from batch 3 performed worse in all aspects, except perhaps with regard to pore-size uniformity. Due to the poor expansion of these foams only the temperature was varied while the rotational speed was kept fixed at $80 \mathrm{rpm}$. The poor foaming was a bit surprising since sodium bicarbonate in combination with citric acid, has been shown to result in a greater expansion than sodium bicarbonate alone in some other studies. Fasihi et al. investigated the 

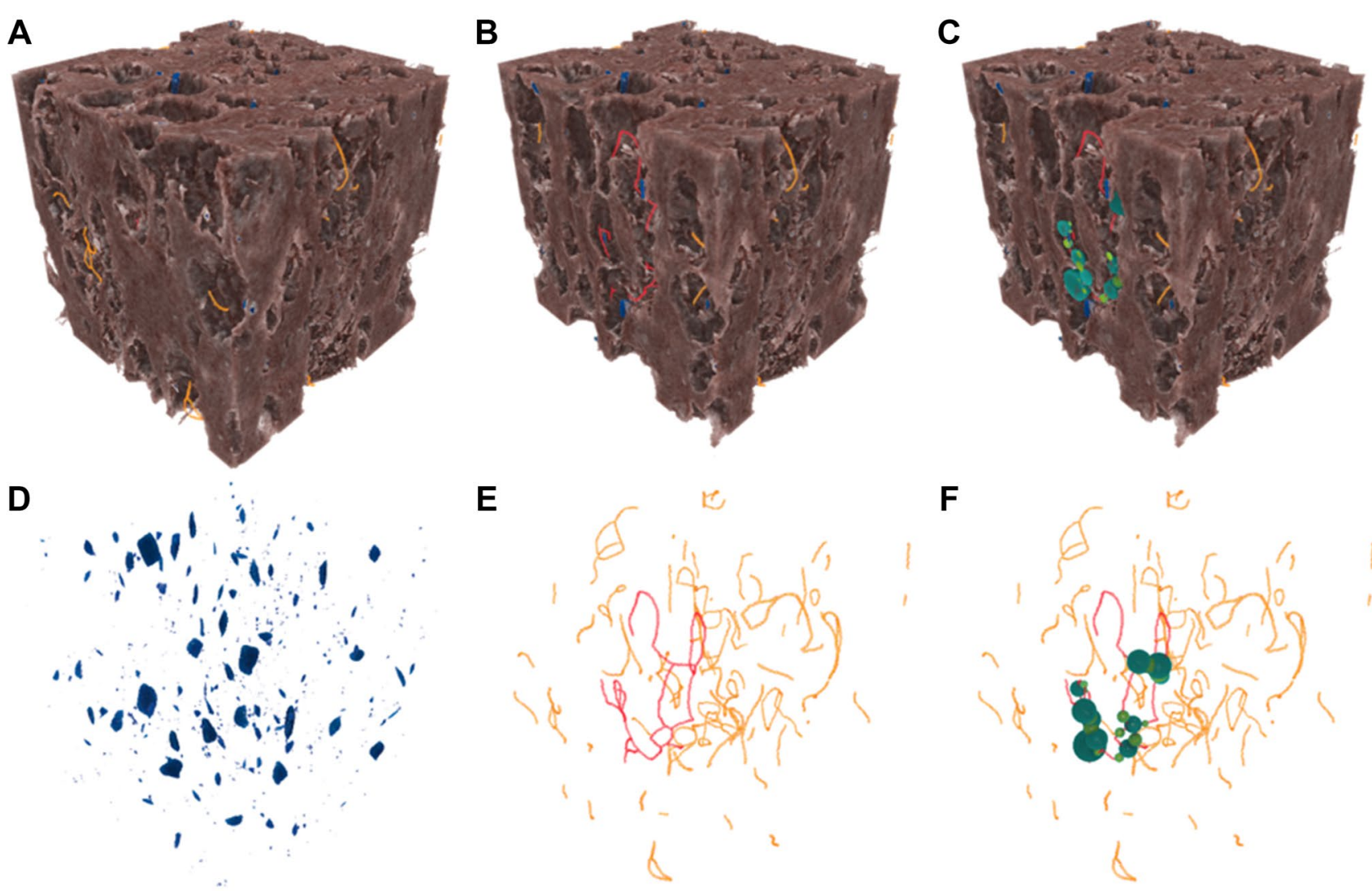

Fig. 5 3D volume rendering of sample B2-6 consisting of a extruded $\beta$-glucan (in brown) and the longest connected image skeleton (in red), as well as isolated non-connected image skeletons (in orange), b vitual cut plane at the longest skeleton, $\mathbf{c}$ maximal inscribed blobs in the porous part along the longest skeleton, $\mathbf{d}$ high absorbing part (in blue), $\mathbf{e}$ image skeletons and $\mathbf{f}$ image skeletons and inscribed blobs at throats (light green) and pores (dark green). The size of the VOI was $1.26 \times 1.26 \times 1.26 \mathrm{~mm}^{3}$. (Color figure online)

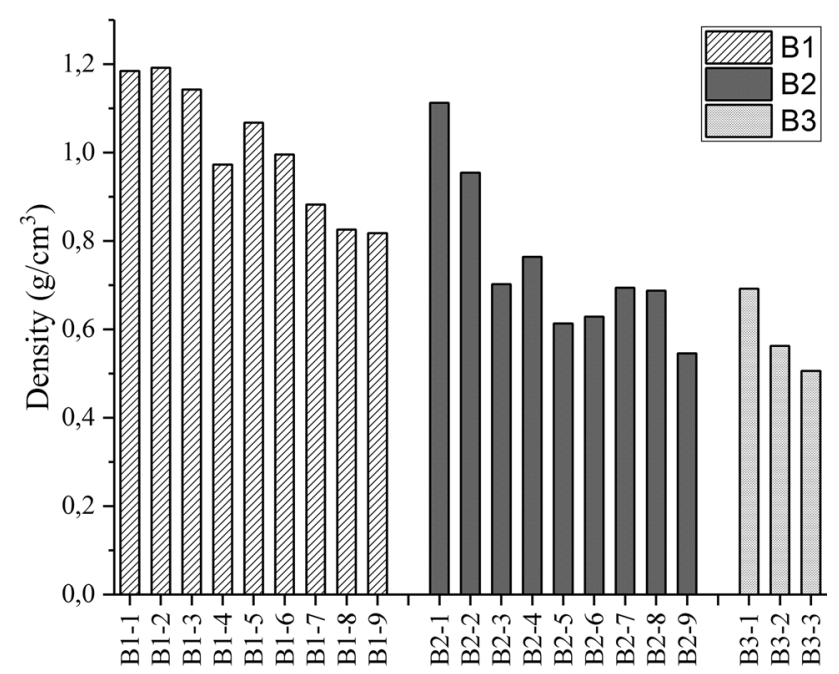

Fig. 6 The apparent density for all the 21 foamed samples. The lowest obtained density was around $0.55 \mathrm{~g} / \mathrm{cm}^{3}$, whereas the more dense samples had values around $1.2 \mathrm{~cm}^{3}$ expansion of polypropylene (PP) samples and concluded that a combination of sodium bicarbonate and citric acid at a ratio of $56 / 44 \%$ by weight resulted in a greater expansion than sodium bicarbonate alone [19]. The exact degradation temperatures of sodium bicarbonate and citric acid are not easily obtained. Sodium bicarbonate has a broad decomposition temperature range and Soares and Nachtigall found the onset temperature of sodium bicarbonate to be as low as $116{ }^{\circ} \mathrm{C}$, but with a maximum decomposition rate at $180{ }^{\circ} \mathrm{C}$ [20]. Some studies report decomposition temperatures of combined blowing agent systems of sodium bicarbonate and citric acid being in the range of $150-200{ }^{\circ} \mathrm{C}[21,22]$, whereas Scholz gave a decomposition temperature of citric acid between 153 and $170{ }^{\circ} \mathrm{C}$ [23], Ruiz et al. found the onset temperature of degradation to be as high as $190-220^{\circ} \mathrm{C}$ [24]. Therefore, in the event of the citric acid probably not being activated at $150{ }^{\circ} \mathrm{C}$, the higher temperature of $170{ }^{\circ} \mathrm{C}$ was also tested for batch 3 , but with a limited effect.

The exact influence of the citric acid on the foaming of the system is at this point not fully understood. Wright and Frank reported on a similar encounter when 
they investigated the effect of different blowing agents on poly(hydroxybutyrate-co-valerate) (PHBV) foaming. Citric acid was eliminated early in the experimental phase due to its lack of gas production and due to a thermal degradation of the PHBV at $160{ }^{\circ} \mathrm{C}$ [25]. One explanation for the lack of expansion when adding the citric acid could therefore be that the decomposition temperature of the substance is above where thermal degradation of the $\beta$-glucan concentrate starts. This does however not explain the hindering of the foaming by the sodium bicarbonate and water at lower temperatures.

On the other hand, a cross-linking effect of citric acid on certain starch-based systems has been reported on previously [26-28]. The cross-linking could take place at quite low temperatures, around $70{ }^{\circ} \mathrm{C}$ [27]. There is thus a possibility that the citric acid and the starch fraction in the $\beta$-glucan concentrate have formed cross-links which hinders the expansion of the material.

Another possible explanation for the lower expansion of foams produced with material from batch 3 could simply be the increased blowing agent content. The increased filler content, which, if not activated, could lead to a reduction of the cell wall extensibility. A similar trend was observed for starch-based systems where an adequate addition of sodium bicarbonate produced a more uniform expansion of the foams, whereas a high addition of sodium bicarbonate led to shrinkage of the material upon exiting the extruder, due to a lowered chain mobility [29].

\section{Conclusions}

It was possible to produce foamed materials from the $\beta$-glucan concentrate by extrusion. The samples had a maximum expansion of about $160 \%$, using only water as the blowing agent. By adding $5 \mathrm{wt}-\%$ sodium bicarbonate, the expansion could be increased to about $200 \%$ and at the same time the size and the spatial distribution of the pores became more uniform. Adding citric acid to the compound significantly reduced the expansion of the foams. This can perhaps be due to a high decomposition temperature of citric acid, exceeding the degradation temperature of the $\beta$-glucan concentrate, in combination with a reduction of the extensibility of the cell walls and a crosslinking of the compound. The most probable scenario is that multiple of the above mentioned effects influenced and hindered the foaming of samples containing citric acid.

Acknowledgements The funding from the Swedish Research Council Formas is gratefully acknowledged. Further thanks are extended to the members of SmartFoam project for supporting this research. Assoc. Prof. Stephen Hall at the 4D Imaging Lab, Lund University, is acknowledged for performing the X-ray microtomography scans and Mrs Annika Altskär for the help with the confocal laser scanning microscopy analysis.

Open Access This article is distributed under the terms of the Creative Commons Attribution 4.0 International License (http://creativeco mmons.org/licenses/by/4.0/), which permits unrestricted use, distribution, and reproduction in any medium, provided you give appropriate credit to the original author(s) and the source, provide a link to the Creative Commons license, and indicate if changes were made.

\section{References}

1. Eriksen M, Lebreton LCM, Carson HS, Thiel M, Moore CJ, Borerro JC, Galgani F, Ryan PG, Reisser J (2014) Plastic pollution in the world's oceans: more than 5 trillion plastic pieces weighing over 250,000 tons afloat at sea PLoS ONE. 9:12

2. Wilcox C, Sebille EV, Hardesty BD (2015) Threat of plastic pollution to seabirds is global, pervasive, and increasing. Proc Natl Acad Sci 112(38):11899-11904

3. Chae Y, An Y (2018) Current research trends on plastic pollution and ecological impacts on the soil ecosystem: a review. Environ Pollut 240:387-395

4. Ahmad A, Anjum FM, Zahoor T, Nawaz H, Dilshad SMR (2012) Beta glucan: a valuable functional ingredient in foods. Crit Rev Food Sci Nutr 52(3):201-212

5. Othman RA, Moghadasian MH, Jones PJ (2011) Cholesterollowering effects of oat $\beta$-glucan. Nutr Rev 69(6):299-309

6. Wolever TMS, Gibbs AL, Brand-Miller J, Duncan AM, Hart V, Lamarche B, Tosh SM, Duss R (2011) Bioactive oat-glucan reduces LDL cholesterol in Caucasians and non-Caucasians. Nutr J 10(1):130-134

7. Tiwari U, Cummins E (2011) Meta-analysis of the effect of [beta]glucan intake on blood cholesterol and glucose levels. Nutrition 27(10):1008-1016

8. Karlsson K, Berta M, Öhgren C, Stading M, Rigdahl M (2018) Flow behaviour and microstructure of a $\beta$-glucan concentrate. $\mathrm{J}$ Polym Environ 26(8):3352-3361

9. Schindelin J, Arganda-Carreras I, Frise E, Kaynig V, Longair M, Pietzsch T, Preibisch S, Rueden C, Saalfeld S, Schmid B, Tinevez J-Y, James White D, Hartenstein V, Eliceiri K, Tomancak P, Cardona A (2012) Fiji: an open-source platform for biological-image analysis. Nat Methods 9(7):676-682

10. Brun F, Mancini L, Kasae P, Favretto S, Dreossi D, Tromba G (2010) Pore3D: a software library for quantitative analysis of porous media. Nucl Instrum Methods Phys Res A 615(3):326-332

11. Bear J (2013) Dynamics of fluids in porous media. Courier Corporation, New York

12. Brun F, Dreossi D (2010) Efficient curve-skeleton computation for the analysis of biomedical 3d images-biomed 2010. Biomed Sci Instrum 46:475-480

13. Ajay L (2012) Drishti: a volume exploration and presentation tool. Developments in X-ray Tomography VIII. International Society for Optics and Photonics, Vol. 8506. https://github.com/nci/drish ti

14. Biron M (2013) Chapter 4-detailed accounts of thermoplastic resins. In: Thermoplastics and thermoplastic composites, 2nd edn. William Andrew, Oxford, pp 189-714

15. Calvert K, Trumble K, Webster T, Kirkpatrick L (2010) Characterization of commercial rigid polyurethane foams used as bone analogs for implant testing. J Mater Sci Mater Med 21(5):1453-1461

16. Mulky T, Niemeyer, K (2018) Computational study of the effects of density, fuel content, and moisture content on smoldering 
propagation of cellulose and hemicellulose mixtures. https://doi. org/10.1016/j.proci.2018.06.164

17. Youssefian S, Rahbar N (2015) Molecular origin of strength and stiffness in bamboo fibrils. Sci Rep. https://doi.org/10.1038/srep1 1116

18. Awal A, Rana M, Sain M (2015) Thermorheological and mechanical properties of cellulose reinforced PLA bio-composites. Mech Mater 80(A):87-95

19. Fasihi M, Targhi AA, Bayat H (2016) The simultaneous effect of nucleating and blowing agents on the cellular structure of polypropylene foamed via the extrusion process. E-Polymers 16(3):235-241

20. Soares FA, Nachtigall SMB (2013) Effect of chemical and physical foaming additives on the properties of PP/wood flour composites. Polym Testing 32(4):640-646

21. Szegda D, Duangphet S, Song J, Tarverdi K (2014) Extrusion foaming of PHBV. J Cell Plast 50(2):145-162

22. Lee CH, Lee K, Jeong HG, Kim SW (2000) Growth of gas bubbles in the foam extrusion process. Adv Polym Technol 19(2):97-112

23. Scholz D (2013) Forty years formulated endothermics in the foamed plastics world. Blowing Agents and Foaming Processes 2012. Proceedings of the 14th International Conference held Berlin, Germany, 8-9 May 2012. Cellular Polymers 32(1):70

24. Ruiz JAR, Vincent M, Agassant J, Sadik T, Pillon C, Carrot C (2015) Polymer foaming with chemical blowing agents: Experiment and modeling. Polym Eng Sci 55(9):2018-2029
25. Wright ZC, Frank CW (2014) Increasing cell homogeneity of semicrystalline, biodegradable polymer foams with a narrow processing window via rapid quenching. Polym Eng Sci 54(12):2877-2886

26. Ghanbarzadeh B, Almasi H, Entezami A (2011) Improving the barrier and mechanical properties of corn starch-based edible films: effect of citric acid and carboxymethyl cellulose. Ind Crops Prod 33:229-235

27. Menzel C, Olsson E, Plivelic T, Andersson R, Johansson C, Kuktaite R, Järnström L, Koch K (2013) Molecular structure of citric acid cross-linked starch films. Carbohyd Polym 96:270-276

28. Olsson E, Hedenqvist M, Johansson C, Järnström L (2013) Influence of citric acid and curing on moisture sorption, diffusion and permeability of starch films. Carbohyd Polym 94:765-772

29. Abinader G, Lacoste C, Baillif ML, Erre D, Copinet A (2015) Effect of the formulation of starch-based foam cushions on the morphology and mechanical properties. J Cell Plast 51(1):31-44

Publisher's Note Springer Nature remains neutral with regard to jurisdictional claims in published maps and institutional affiliations. 